Received: 2017.12.21

Accepted: 2018.01.08

Published: 2018.07 .03
Authors' Contribution:

Study Design A

Data Collection B

Statistical Analysis C

Data Interpretation D

Manuscript Preparation E

Literature Search F

Funds Collection G

\title{
Long Noncoding RNA LINC003121 Inhibits Proliferation and Invasion of Thyroid Cancer Cells by Suppression of the Phosphatidylinositol- 3-Kinase (PI3K)/Akt Signaling Pathway
}

\author{
Xiang Min \\ Kai Liu \\ Hengtao Zhu \\ Jian Zhang
}

Department of Otorhinolaryngology and Head and Neck Surgery, The First Affiliated Hospital of Nanchang University, Nanchang, Jiangxi, P.R. China
Corresponding Author: Source of support:
Jian Zhang, e-mail: zhangjianj1028@163.com

Departmental sources

Background: The aim of this study was to explore the potential effects of long noncoding RNA (IncRNA) LINC003121 on thyroid cancer (TC) cell proliferation and invasion and to explore their possible mechanisms with the involvement of the PI3K/Akt signaling pathway.

Material/Methods: We enrolled 211 thyroid cancer tissues and 70 adjacent normal tissues in this study. TC cell lines K1, SW579, and $8505 \mathrm{C}$ and the human thyroid follicular cell line Nthy-ori3-1 were selected and assigned into blank, control vectors, LINC00312 vectors, si-control, and si-LINC00312 groups. Quantitative real-time PCR was used to determine the levels of LINC003121 and Western blotting was used to detect the protein expression of MMP-9, PI3K, t-Akt, and p-Akt. Cell proliferation was assessed by CCK8 assay and EdU incorporation assay, and cell invasion was assessed by Transwell assay.

Results: The expression of LINC00312 was significantly decreased in TC tissues and cell lines. In an in vitro experiment, si-LINC00312 significantly promoted the invasion and proliferation of TC cells. Conversely, overexpression of LINC00312 decreased cell proliferation and invasion in vitro, and decreased tumorigenicity in TC xenograft models in nude mice. LINC00312-mediated tumor suppression in TC cells may occur via suppression of activation of the PI3K/Akt signaling pathway and expression of MMP-9, and the role of MMP-9 expression induced by overexpressed LINC00312 or si-LINC00312 could be weakened by LY294002 (PI3K inhibitor).

Conclusions: LINC00312 can act as a tumor-suppressor in TC by attenuating the PI3K/Akt signaling pathway, and LINC00312 could be a novel diagnosis biomarker and a promising therapeutic target for TC patients.

MeSH Keywords: $\quad$ Cell Proliferation • Neoplasm Invasiveness • Parathyroid Neoplasms • Proto-Oncogene Proteins c-akt - RNA, Long Noncoding

Full-text PDF: https://www.medscimonit.com/abstract/index/idArt/908652

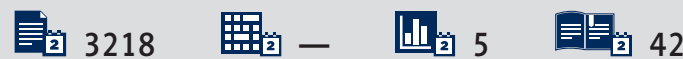




\section{Background}

Thyroid cancer (TC) is a common endocrine malignancy, and contains a variety of different histological types, including differentiated TC, undifferentiated (anaplastic) TC, and medullary TC [1]. The incidence rates of TC have been increasing worldwide, and it can affect males and females at all ages, with the most rapid increase found among women of reproductive age [2,3]. Women are more likely to have TC than men, with a female: male sex ration of 3: 1 [4]. Risk factors that increase the incidence of TC can be divided into 2 main types: exogenous (e.g., exposure to ionizing radiation, iodine intake, westernized lifestyle, and environmental pollutants) and endogenous (e.g., thyroid-stimulating hormone levels, autoimmune, thyroiditis, obesity, and insulin resistance) [5]. TC is usually asymptomatic and is often incidentally found in routine examinations, and only about $5 \%$ of nodules are malignant; however, $3 \sim 15 \%$ of TC patients show distant metastases at diagnosis, and another $6 \sim 20 \%$ develop distant metastases during followup [6,7]. Although TC usually has an excellent prognosis, few treatment regimens are available [8] and new treatment targets are urgently needed.

Long noncoding RNAs (IncRNAs) are endogenous cellular RNAs (>200 nucleotides in length) that lack an open reading frame of significant length [9]. A recent report revealed that the abnormal expression of IncRNAs results in tumor-suppressive and pro-tumor effects in different types of cancer [10]. Additionally, increasing evidence demonstrates that IncRNAs modulate a number of cellular functions, including cell growth, differentiation, cell cycle progression, and apoptosis [11]. Long intergenic noncoding RNA LINC00312 (LINC00312), also referred to as NAG7, is a long intergenic noncoding RNA located on 3p25.3 and has been proven to be a novel putative tumor suppressor gene in many cancers [12]. A previous study also confirmed that LINC00312 can serve as a potential biomarker for progression, metastasis, and prognosis in nasopharyngeal carcinoma [13]. The phosphoinositide 3-kinase (PI3K)/Akt pathway was found to play a fundamental role in the tumorigenesis and aggressiveness of human cancers, including TC, prostate cancer, and gastric cancer [14-16]. Subsequently, this signaling pathway also has been established to have important roles in regulating cell proliferation, differentiation, division, and apoptosis, and, when disordered, it promotes tumorigenesis [17]. Previous studies demonstrated that genetic alterations are common in many components of the PI3K/Akt signaling pathway in TC, and the PI3K/Akt signaling pathway is an attractive therapeutic target for TC $[18,19]$. Therefore, the relationship between the PI3K/Akt signaling pathway and TC is well-established, but the exact relationship between LINC00312 and the PI3K/Akt signaling pathway in TC remains to be explored.

\section{Material and Methods}

\section{Ethical statement}

The study was approved by the Ethics Committee of the First Affiliated Hospital of Nanchang University. Informed consent forms were signed by all enrolled patients.

\section{Study subjects}

Between October 2013 and August 2015, 211 patients (99 females and 112 females) diagnosed with TC and who underwent primary surgical resection at the First Affiliated Hospital of Nanchang University were enrolled in our study collecting 211 TC tissues and 70 adjacent normal tissues ( $2 \mathrm{~cm}$ away from the tumor site). All the collected samples were confirmed by pathological examination. Before enrollment, none of the patients had received any TC treatment and had no severe systemic diseases such as malignant tumors or severe systemic infections. The collected samples were stored at $-70^{\circ} \mathrm{C}$ for further use.

\section{Cell culture}

K1 (papillary TC), SW579 (squamous TC), and 8505C (anaplastic TC) cell lines were purchased from the Chinese Academy of Sciences (Shanghai, China) and were cultured in Dulbecco's modified Eagle's medium (DMEM) (Promega, Madison, WI) containing 15\% FBS (HyClone, Logan, UT) and $100 \mathrm{U} \cdot \mathrm{mL}^{-1}$ streptomycin sulfate (Invitrogen-Gibco). The human thyroid follicular cell line Nthy-ori3-1 was purchased from ATCC (Manassas, VA, USA) and was incubated in RPMI Media1640 (InvitrogenGibco) containing $10 \% \mathrm{FBS}, 100 \mathrm{U} \cdot \mathrm{mL}^{-1}$ penicillin $\mathrm{G}$ sodium salt and $100 \mathrm{U} \cdot \mathrm{mL}^{-1}$ streptomycin sulfate. The above cells were all incubated at $5 \% \mathrm{CO}_{2}$ in an incubator with $95 \%$ relative humidity at $37^{\circ} \mathrm{C}$. When cells reached $80 \%$ confluency, sub-culturing was conducted. After that, cells were washed 2 times with PBS and digested with trypsin. The trypsin was discarded when the intercellular space was enlarged. Cells were passaged without suspension in the culture medium.

\section{Cell transfection}

LINC00312 siRNA and negative control (NC) siRNA were constructed by Sangon Biotech (Shanghai, China). The sequences of si-LINC00312 were: sense, 5'-CCCAGGUACUAAGUUAUGUTT-3' and antisense, 5'-ACGUGACACGUUCGGAGAATT-3', and the sequences of si-NC were: sense, 5'-UUCUCCGAACGUGUCACGUTT-3' and antisense, 5'-ACGUGACACGUUCGGAGAATT-3'. According to the NCBI Reference Sequence, NR_024065.2, LINC00312 overexpression plasmid was constructed. pcDNA3.1 vector was used as plasmid vector and empty control (Invitrogen, Shanghai, China). Cells were cultured in 6-well plates, and according to 
the instructions, siRNA or NC were transfected into cells using Lipofectamine 2000 (Invitrogen, USA), and plasmid vectors were transfected into cells using X-treme GENE HP DNA transfection reagent (Roche, Basel, Switzerland). After 48 h, cells were harvested for the following experiments.

\section{Quantitative real-time polymerase chain reaction (qRT-PCR)}

Total RNA was extracted from TC tissues and adjacent normal tissues using the TRIzol method (Invitrogen, Carlsbad, CA, USA). The obtained RNA was dissolved in $0.1 \%$ diethylpyrocarbonate water (Fermentas, Glen Burnie, MD, USA) and then optical density (OD) at 260/280 nm and RNA quality were detected. Reverse transcription and PCR were also conducted in accordance with the instructions. Reverse transcription was performed as follows: $10 \mathrm{~min}$ at $74^{\circ} \mathrm{C}, 2 \mathrm{~min}$ of ice bathing, $60 \mathrm{~min}$ at $42^{\circ} \mathrm{C}$, and $10 \mathrm{~min}$ at $70^{\circ} \mathrm{C}$. The reaction conditions were: pre-denaturation at $95^{\circ} \mathrm{C}$ for $1 \mathrm{~min}$, and 40 cycles of at $95^{\circ} \mathrm{C}$ for $15 \mathrm{~s}$, at $60^{\circ} \mathrm{C}$ for $30 \mathrm{~s}$, and at $72^{\circ} \mathrm{C}$ for $30 \mathrm{~s}$. The relative expression of target genes was calculated using the $2^{-\Delta \Delta c t}$ method with $\beta$-actin as the internal reference. Each experiment was conducted 3 times. Primer sequences for LINC00312 and $\beta$-actin were: sense: 5'-AAGCGAACCAAGCCAATA-3' and antisense: 5'-CAAATCCCTGAAACTCTG-3'; sense: 5'-CAG CAAGCAGGAGTATGACG-3' and antisense: 5'-GAAAGGGTG TAACGCAACTAA-3'.

\section{Cell counting kit (CCK)8 assay}

$\mathrm{K} 1, \mathrm{SW} 579$, and $8505 \mathrm{C}$ cells in logarithmic growth phase were digested with trypsin and the cell concentration was adjusted. Cells were seeded into a 6 -well plate with $1 \times 10^{5}$ cells/well, cultured in an incubator for $24 \mathrm{~h}$, and then transfected. After that, the cells were digested with trypsin and the cell culture medium was collected and placed into centrifuge tubes. The cells were then seeded in 496 -well plates (3000 cells/well) when the cell concentration reached $3 \times 10^{5} / \mathrm{ml}$. Four groups were set in each plate with 4 replicates set in each group. The samples were incubated in culture plates with $5 \% \mathrm{CO}_{2}$ at $37^{\circ} \mathrm{C}$ in an incubator. The plates were taken out after $24,48,72$, and $96 \mathrm{~h}$ of incubation. CCK8 solution ( $10 \mu \mathrm{l}$, Sigma, SF, USA) was added into each well (avoiding bubbles). The culture plates were placed in an incubator at $37^{\circ} \mathrm{C}$ with $5 \% \mathrm{CO}_{2}$ for $2 \mathrm{~h}$. The $O D$ value was measured at $450 \mathrm{~nm}$ using a microplate reader (Bio-Rad, Cal, USA). The experiment was repeated 3 times.

\section{5-ethynyl-2'-deoxyuridine (EdU) incorporation assay}

After transfection for $48 \mathrm{~h}$, EdU incorporation assay was performed in K1, SW579, and 8505C cells using the Cell-Light EdU imaging detecting kit (RiboBio, Guangzhou, China) according to the instructions. As an alternative thymidine analog, incorporation of EdU can label and identify cells undergoing DNA replication. EdU-positive cells were calculated with the formula $=($ EdU add-in cells $/$ Hoechst stained cells $) \times 100 \%$.

\section{Transwell assay}

Matrigel $(30 \mu \mathrm{l})$ was dissolved overnight and diluted with FBSfree DMEM in triplicate volume. After that, Matrigel was added into each well in the upper chamber (added at a 15-min interval). Each well in the upper chamber was inoculated with $2 \times 10^{4}$ cells. DMEM $(0.5 \mathrm{ml})$ containing $10 \% \mathrm{FBS}$ was added to each well of the lower chamber. After $24 \mathrm{~h}$ of incubation, the cells in each well were fixed with paraformaldehyde and stained with $0.1 \%$ Crystal Violet for $0.5 \mathrm{~h}$ to remove the uninfected cells from the upper chamber. Next, cells were washed with 0.1M PBS. Fields were randomly selected for counting and photographing cells under a light microscope. The experiment was repeated 3 times. The cells passing through the Matrigel were analyzed to determine the invasion ability.

\section{Inhibition effect of LY294002 on PI3K/Akt in cells}

$\mathrm{K} 1$, SW579, and $8505 \mathrm{C}$ cells were inoculated into a 6-well plate and incubated at $37^{\circ} \mathrm{C}$ overnight. After that, the cells were treated with $25 \mu \mathrm{mol} / \mathrm{L}$ LY294002 (San Diego, CA), a phosphatidylinositol 3'-kinase (PI3K) inhibitor for $72 \mathrm{~h}$, followed by transfection of lentivirus.

\section{Western blotting}

Protein was separated by $12 \%$ sodium dodecyl sulfate-polyacrylamide gel electrophoresis and transferred into nitrocellulose membranes. Membranes were incubated with the primary antibodies anti-MMP9 (Abcam), anti-PI3K (Abcam), anti-p-Akt, and anti-t-Akt (Abcam) at $4^{\circ} \mathrm{C}$ overnight. Then, membranes were washed and incubated for $2 \mathrm{~h}$ with the addition of horseradish peroxidase (HRP)-labeled anti-rabbit secondary antibodies (Prosci Inc., Poway, CA). Subsequently, electrochemiluminescence reagents (Pierce antibodies; Thermo Fisher Scientific) were added for detection and visualization.

\section{Immunohistochemistry}

The $8505 \mathrm{C}$ tumors were formalin-fixed, embedded in paraffin, and cut into $6-\mu \mathrm{m}$ sections. Antigen retrieval was conducted in $10 \mathrm{mM}$ sodium citrate buffer $(\mathrm{pH} 6)$ at $96-98^{\circ} \mathrm{C}$ for $16 \mathrm{~min}$. After that, the sections were incubated with primary antibodies against MMP2 (Abcam). Subsequently, according to the instructions, sections were incubated with the Cell \& Tissue Staining Kit HRP-DAB system (R\&D Systems, Minneapolis, MN). Immunostaining was conducted with known positive and negative tumor controls and were blindly assessed by a pathologist. 


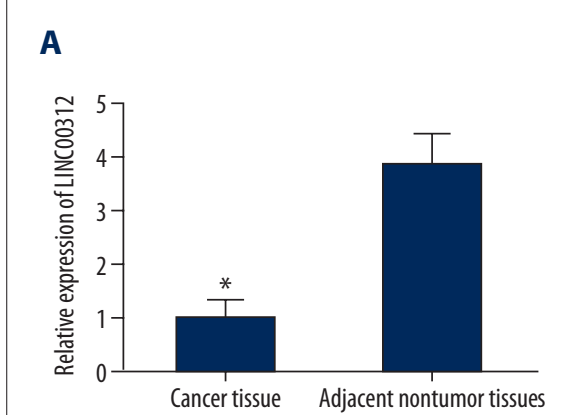

D

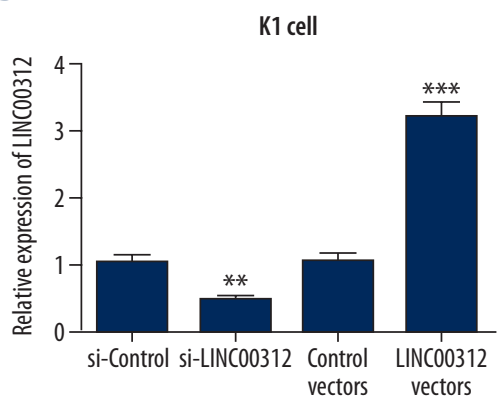

B

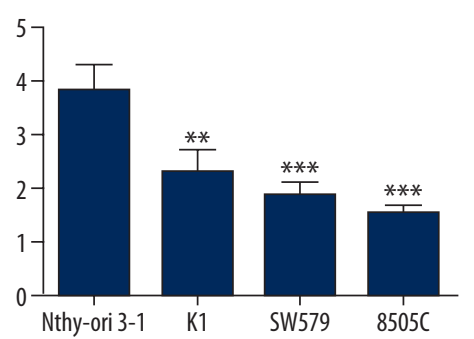

C

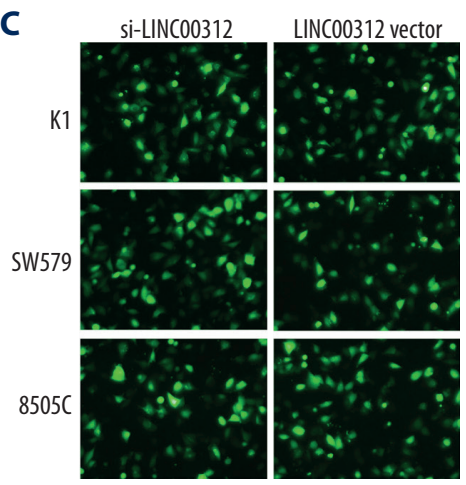

8505 C cell
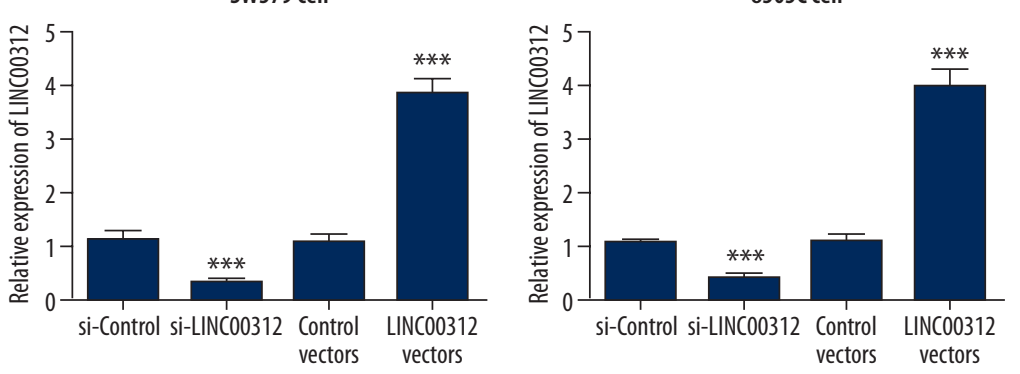

Figure 1. LINC00312 expression in TC cells and tissues. (A) Relative expression of LINC00312 in the TC and adjacent normal tissues; (B) LINC00312 expression in the TC cell lines, K1, SW579, and 8505C, and the normal thyroid cell line, Nthy-ori 3-1; (C) green fluorescent in K1, SW579 and 8505 C cells under fluorescence microscope; (D) detection of transfection efficiency of siLINC00312 or LINC00312 vectors in K1, SW579, and 8505C cells, LINC00312 expression was detected by qRT-PCR. Results presented as the mean $\pm \mathrm{SD}$ with 3 independent experiments. Compared with the si-control group, ${ }^{*} P<0.05,{ }^{* *} P<0.01$ and ${ }^{* * *} P<0.001$. TC - thyroid cancer.

\section{Orthotopic TC xenografts in nude mice}

The $8505 \mathrm{C}$ cells in logarithmic growth phase with stable transfection were digested with trypsin and made into cell suspension. Then, the cells were counted under a microscope, and cells were packaged and stored at $-20^{\circ} \mathrm{C}$ for further use. Healthy nude mice age 6 8 weeks (Hunan SJA Laboratory Animal Co. Ltd, Changsha, China) were selected and grouped into blank, control vectors, LINC00312 vectors, si-control, and si-LINC00312 groups, with 5 mice in each group. The cell suspensions $\left(1 \times 10^{5}\right)$ were successfully injected into each group of cells into the left and right sides of the nude mice. After injection, the mice were raised with conventional method. After the tumors were formed, tumor size (length, width, height) was measured every 2 days and a rough calculation of tumor volume was also performed. The tumor volume was calculated with the formula: $1 / 2 \times$ length $\times$ width $^{2}$. Mice were sacrificed 3 weeks later, and all xenograft tumors were excised, weighed, and collected for immunohistochemistry and Western blotting.

\section{Statistical analysis}

SPSS20.0 software (SPSS, Inc, Chicago, IL) was used for data analysis, and measurement data are expressed by mean \pm standard deviation. Comparisons between 2 groups were conducted by use of the independent-samples $t$ test. Multiple group difference was analyzed by one-way analysis of variance (ANOVA), after which the LSD $t$ test was used for comparison between groups. $P<0.05$ was considered statistically significant.

\section{Results}

\section{LINC00312 was expressed at low level in TC cell lines and TC tissues}

As presented in Figure 1A, LINC00312 expression was significantly decreased in TC tissues when compared with the adjacent normal tissues $(P<0.01)$. Similarly, qRT-PCR demonstrated that the relative expression of LINC00312 was significantly decreased in $\mathrm{K} 1$, SW579, and $8505 \mathrm{C}$ cell lines compared with the human thyroid follicular cell line Nthy-ori3-1 $(P<0.01, P<0.001$ and $P<0.001$, Figure $1 \mathrm{~B})$. To assess the role of LINC00312 in 


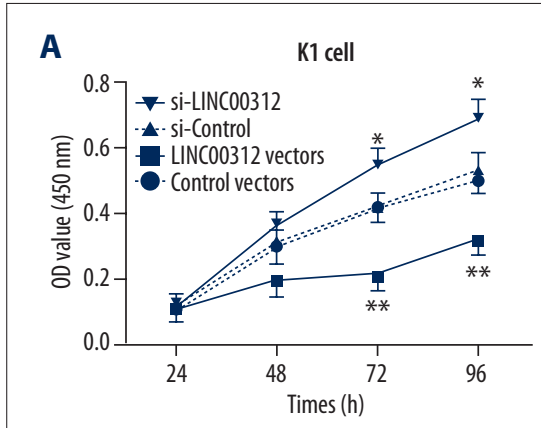

B
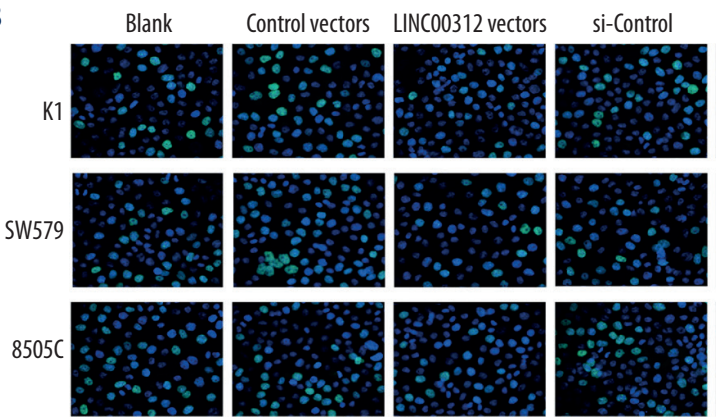
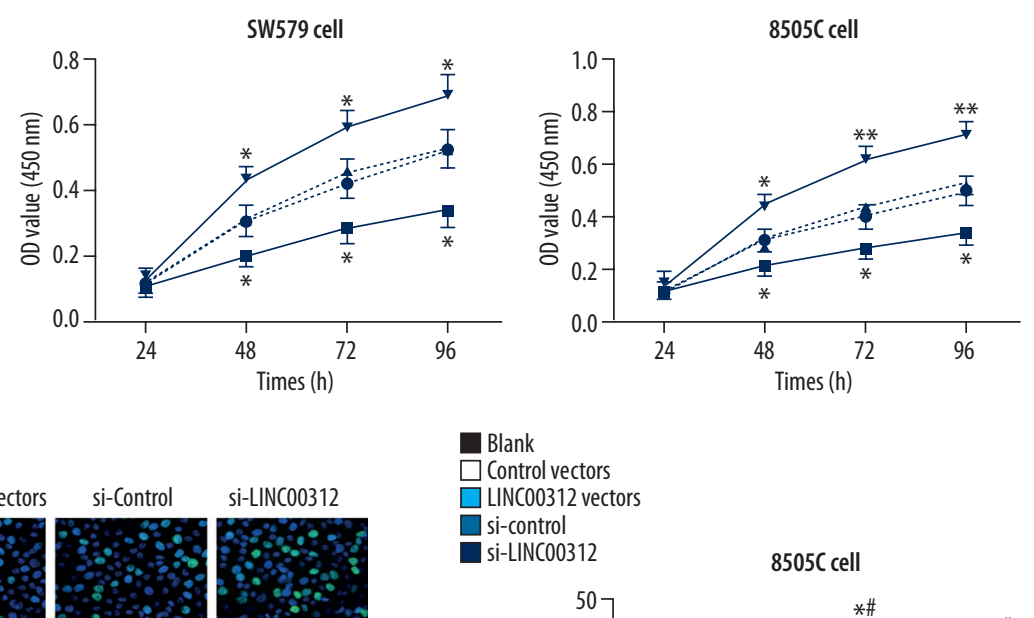

Blank

$\square$ Control vectors

$\square$ LINC00312 vectors

$\square$ si-control

si-LINC00312
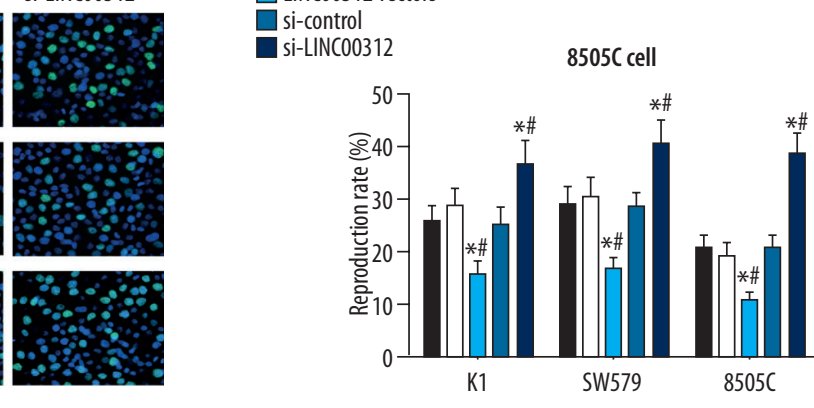

Figure 2. Effect of overexpressed LINC00312 or si-LINC00312 on proliferation of K1, SW579, and 8505C cells. (A) After the upregulation or downregulation of LINC00312, the proliferation ability of transfected or non-transfected K1, sw579, and 8505 c cells was detected by CCK- 8 assay. ${ }^{*}$ Compared with the blank group at the same time point, $P<0.05$; \# compared with si-control group at the same time point, $P<0.05$. (B) After upregulation or downregulation of LINC00312, the proliferation of $\mathrm{K} 1$, sw579, and $8505 \mathrm{c}$ cells was detected by EdU incorporation assay. ${ }^{*}$ Compared with the blank group, $P<0.05$; \# compared with the si-control group, $P<0.05$. Results presented as the mean \pm SD with 3 independent experiments.

these cell lines, si-LINC00312 or an overexpression vector (LINC00312 vectors) were transfected into K1, SW579, and $8505 \mathrm{C}$ cells, and the transfection efficiency was more than 90\% (Figure 1C). Figure 1D revealed that LINC00312 expression in $\mathrm{K} 1$, SW579, and $8505 \mathrm{C}$ cells were decreased by $46.0 \%$ $(P<0.01), 32 \%(P<0.001)$, and $42 \%(P<0.001)$, respectively, after being transfected with si-LINC00312, while LINC00312 expression in K1, SW579, and $8505 \mathrm{C}$ cells was increased by 3.25 times $(P<0.001), 3.88$ times $(P<0.001)$, and 4.02 times $(P<0.001)$, respectively, after transfection with LINC00312 vectors.

\section{The proliferation ability of TC cells was weakened after overexpression of LINC00312}

In vitro, the effect of expression of LINC00312 on proliferation of K1, SW579, and $8505 \mathrm{C}$ cells was detected. CCK-8 assay showed that with the extension of time, the OD value was decreased gradually in cells after overexpression of LINC00312, while the OD value was increased after overexpression of si-LINC00312. Similarly, EdU incorporation assay showed that after overexpression of LINC00312, the proliferation rate of TC cells in the LINC00312 vector group was significantly lower than that in the control vector group $(P<0.05)$, while the proliferation rate of TC cells in the si-LINC00312 group was significantly higher than that in the si-control group after si-LINC00312 overexpression $(P<0.05$, Figure $2 \mathrm{~A}, 2 \mathrm{~B})$. These results demonstrate that LINC00312 can regulate the proliferation of TC cells, the proliferation ability of TC cells was weakened after overexpression of LINC00312, and the proliferation ability of TC cells was enhanced after si-LINC00312 overexpression (Figure 2).

\section{LINC00312 inhibits the invasion ability of TC cells}

Transwell assay was performed to assess the effect of LINC00312 expression on invasion ability of K1, SW579, and $8505 \mathrm{C}$ cells. Results showed that the invasion ability of $\mathrm{K} 1$, SW579, and $8505 \mathrm{C}$ cells was inhibited after overexpression of LINC00312, and it was enhanced after si-LINC00312 overexpression $(P<0.05)$. To further explore the mechanism of by which LINC00312 affects invasion, we used Western blotting to detect the TC invasion-related indicator MMP-9 [20,21]. The results showed that the expression of LINC00312 can significantly affect the expression of MMP-9 $(P<0.05)$, upregulation of LINC00312 reduced the expression of MMP-9 protein, and downregulation of LINC00312 increased the expression of MMP-9 protein (all $P<0.05$ ) (Figure 3). 


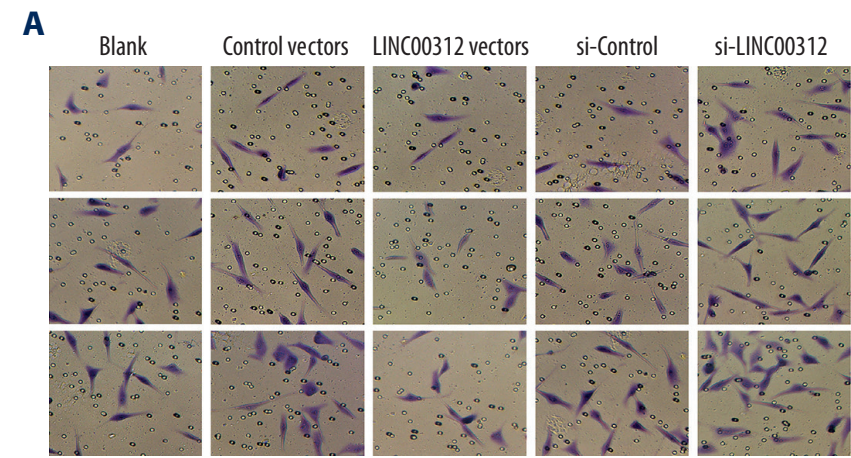

C

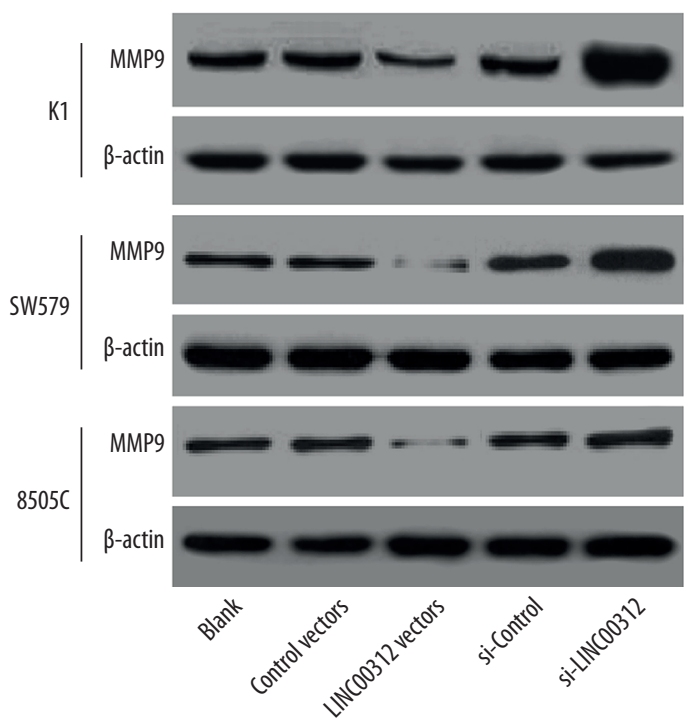

B

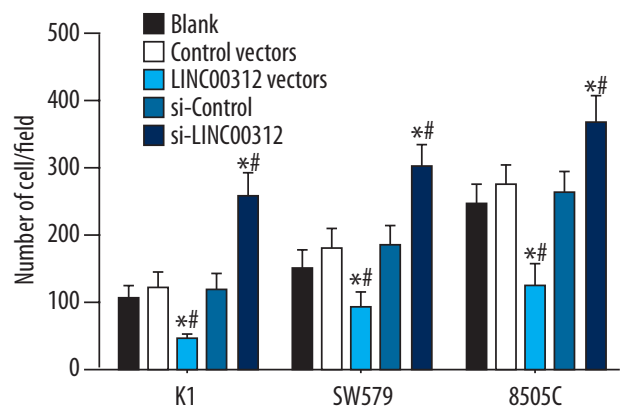

D

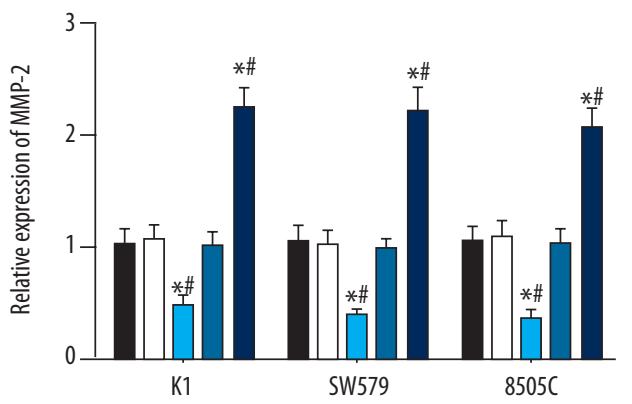

Figure 3. Effect of overexpressed LINC00312 or si-LINC00312 on invasion of the K1, SW579, and 8505C cells. (A, B) The invasive ability of the cells in each group was observed by microscope, and the number of invasive cells in each group was counted. Results showed that after the expression of LINC00312 was increased, the number of cells penetrating the membrane was decreased, but the invasive ability was increased after low expression; (C, D) Western blotting was used to detect the MMP9 protein expression bands and statistical analysis maps, and results revealed that upregulation of LINC00312 reduced the expression of MMP9 protein, and downregulation of LINC00312 increased the expression of MMP9 protein. ${ }^{*}$ Compared with the blank group, $P<0.05$; " compared with the si-control group, $P<0.05$. Results presented as the mean \pm SD with 3 independent experiments.

\section{Overexpression of LINC00312 inhibited the activation of the PI3K/Akt signaling pathway in TC}

To investigate whether the PI3K/Akt signaling pathway is involved in the regulation of LINC00312 on TC cell proliferation and invasion, the expressions of PI3K, t-Akt, and phosphorylated Akt (p-Akt) were detected by Western blotting. Results revealed that overexpression of LINC00312 significantly decreased the expressions of PI3K and $\mathrm{p}$-Akt (both $P<0.05$ ), low expression of LINC00312 significantly increased the expressions of PI3K and p-Akt (both $P<0.05$ ), and overexpression of LINC00312 had no significant effect on t-Akt expression $(P>0.05)$. To further verify the effect of LINC00312 on the PI3K/Akt signaling pathway, we pretreated cells with LY294002 (an inhibitor of the PI3K/Akt signaling pathway). Western blotting results indicated that the effect of MMP-9 expression induced by overexpressed LINC00312 or si-LINC00312 could be weakened by LY294002 ( $P<0.05)$ (Figure 4). Thus, LINC00312 can inhibit the activity of PI3K/Akt signaling pathway in TC cells and inhibits TC invasion to some extent. 

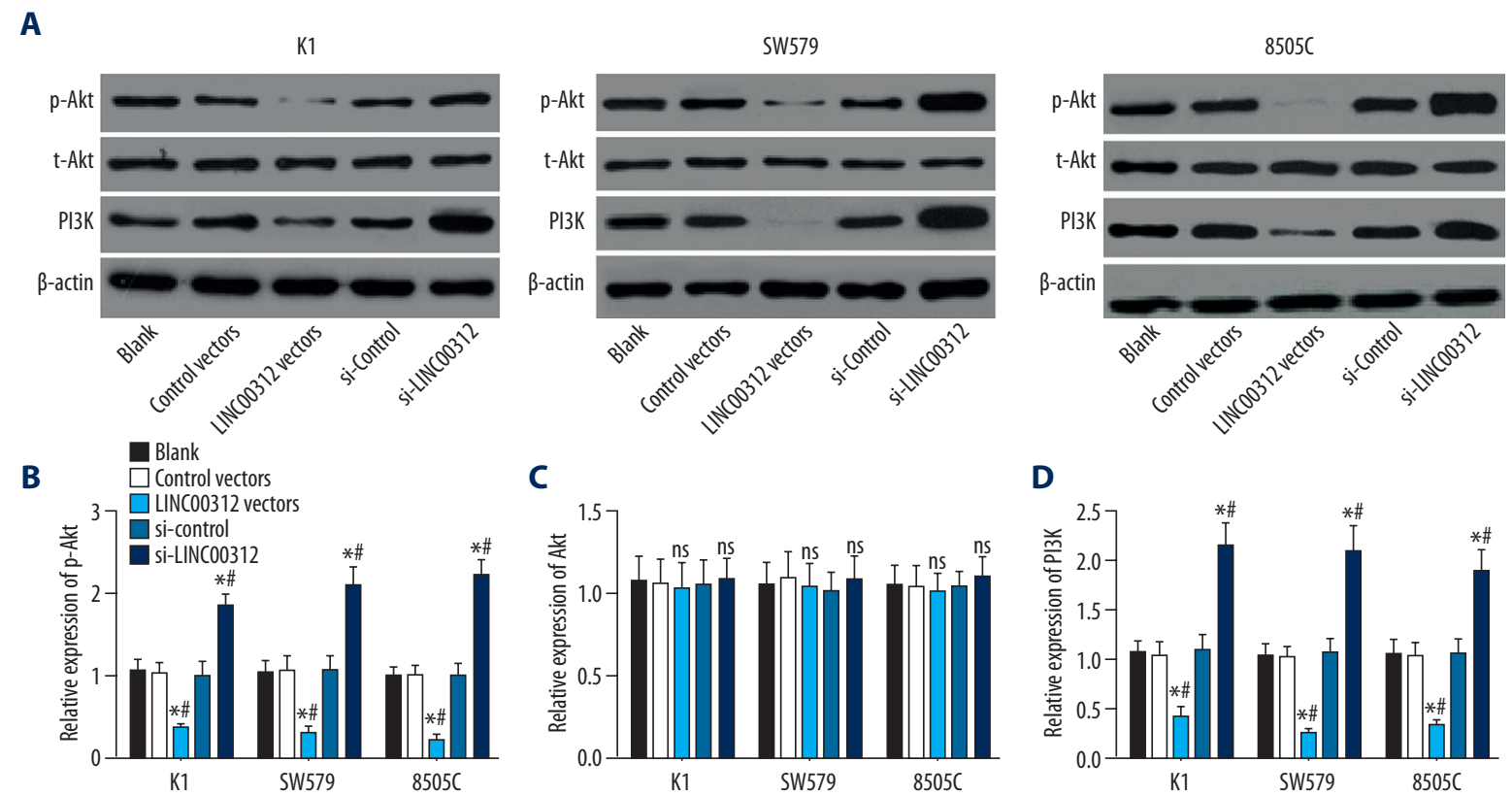

C

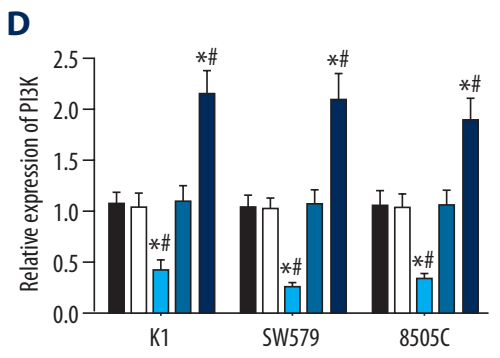

$\mathbf{E}$
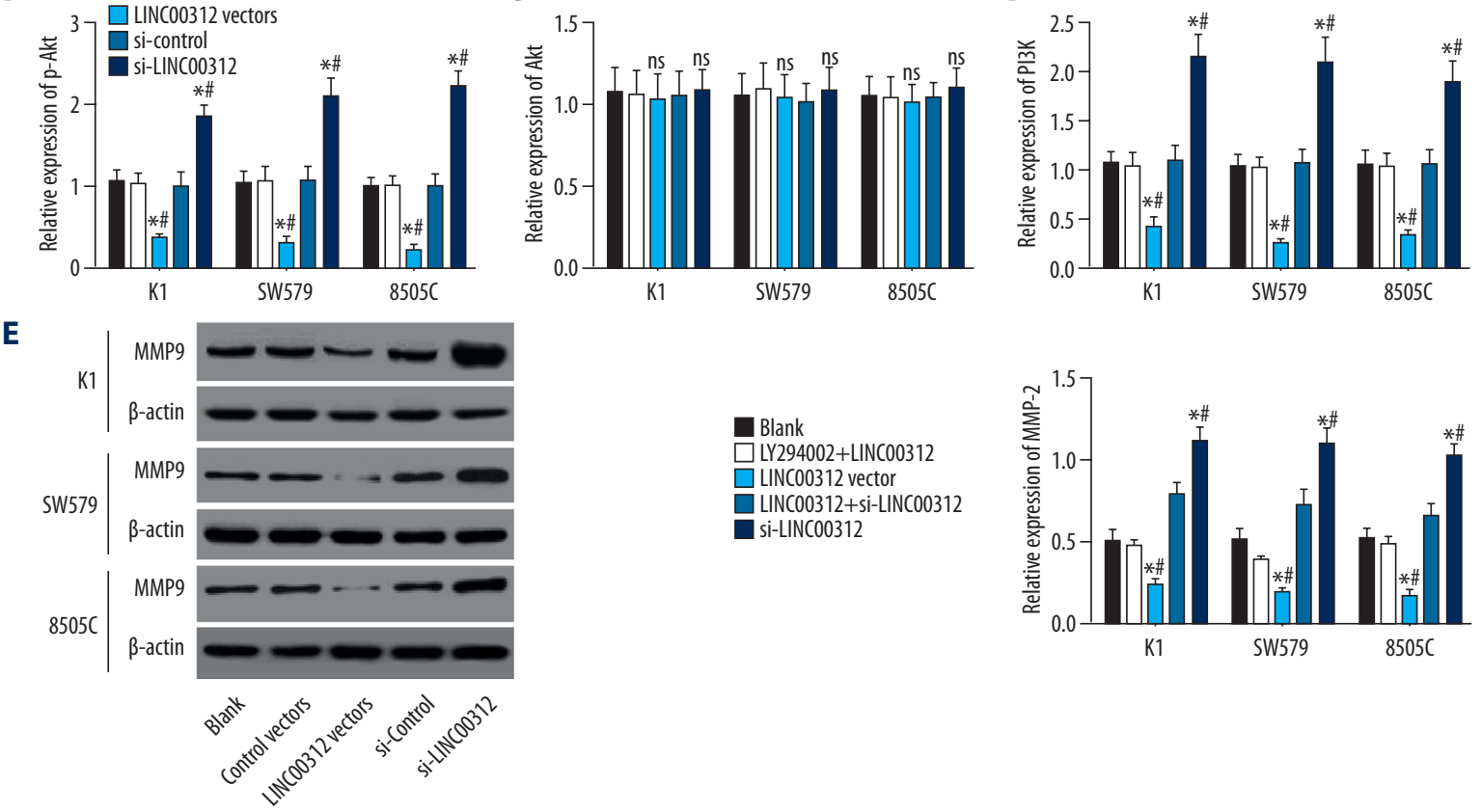

Figure 4. LINC00312 inactivated the PI3K/Akt signaling pathway in TC cell lines. (A-D) The expressions of PI3K, t-Akt, and p-Akt were detected by Western blotting, showing that overexpression of LINC00312 significantly decreased the expressions of PI3K and p-Akt, low expression of LINC00312 significantly increased the expressions of PI3K and p-Akt, and overexpression of LINC00312 had no significant effect on t-Akt expression; (E) The protein expression level of MMP9 was detected by Western blotting, showing that the expression of MMP9 induced by low expression of LINC00312 was weakened after inhibition of the PI3K/Akt signaling pathway. ${ }^{*}$ Compared with the blank group, $P<0.05$; \# compared with the LY294002 +LINC00312 group, $P<0.05$; \& compared with the LY294002 + si-LINC00312 group, $P<0.05$. Results presented as the mean \pm SD with 3 independent experiments.

\section{In vivo experiments demonstrated that LINC00312 inhibited the proliferation and invasion of TC cells}

To determine the effect of LINC00312 on cell proliferation and invasion in vivo, the $8505 \mathrm{C}$ cells with overexpressed LINC00312 or si-LINC00312 and corresponding 8505 C cells in the NC group were inoculated on the ventral side of the forelimb in nude mice. The results demonstrated that compared with the blank group, the growth of subcutaneous tumor with overexpressed LINC00312 was significantly inhibited, while the growth of subcutaneous tumors with si-LINC00312 was significantly increased. Similarly, immunohistochemistry was performed after tumor formation, and the results showed that upregulation of LINC00312 reduced the positive expression of MMP-9 protein and downregulation of LINC00312 increased the positive expression of MMP-9 protein. Finally, protein was extracted from tumor tissues, and Western blotting was performed, showing that the LINC00312 vectors group had decreased expressions of PI3K and p-Akt, while the si-LINC00312 group had increased expressions of PI3K and p-Akt (Figure 5). 


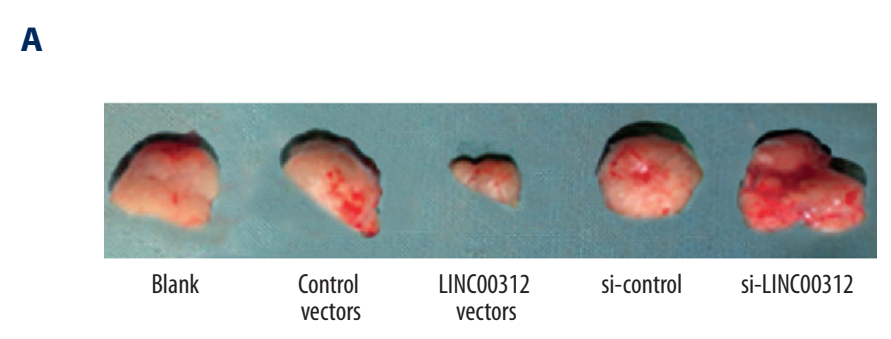

C

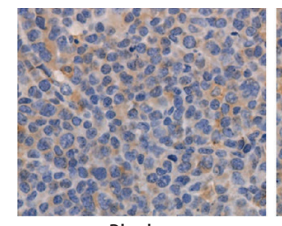

Blank

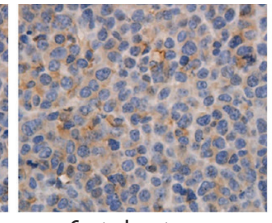

Control vectors

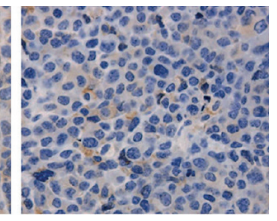

LINC00312 vectors

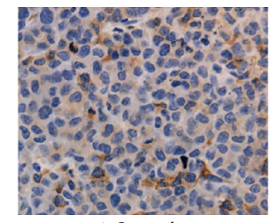

si-Control

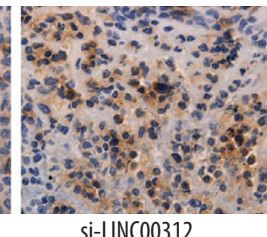

si-LINC00312
E

p-Akt

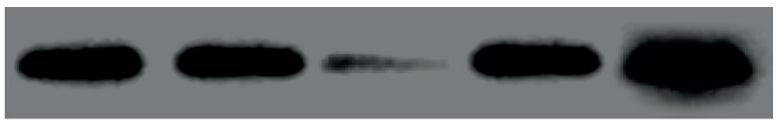

PI3K

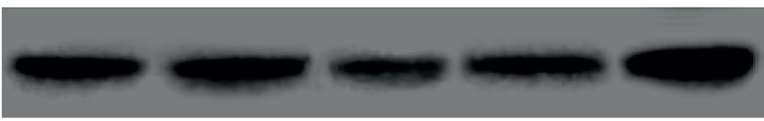

$\beta$-actin

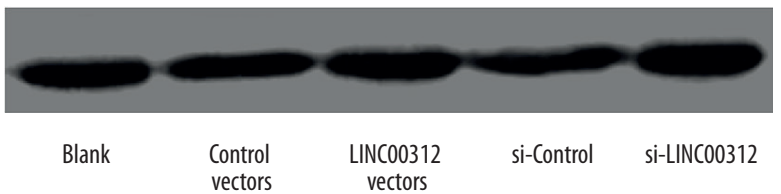

B

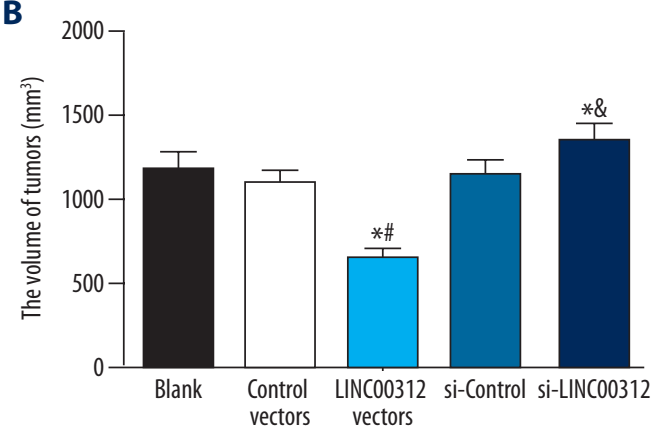

D
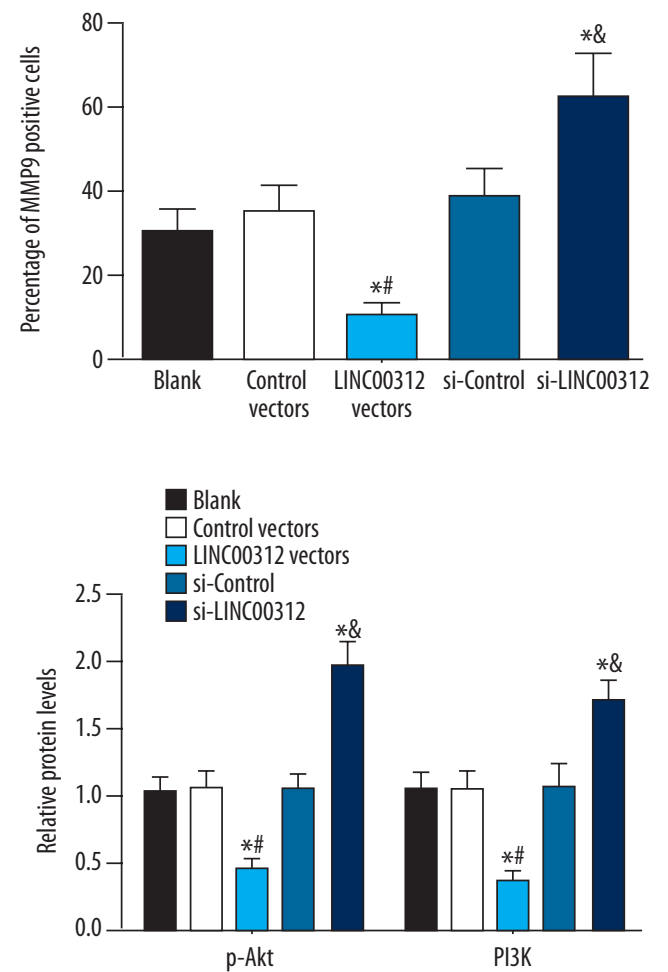

Figure 5. In vivo experiments demonstrated the inhibitory effect of LINC00312 on proliferation and invasion of TC cells.

(A, B) In the subcutaneous model of TC, overexpression of LINC00312 inhibited the growth of TC, but the growth of TC in the si-LINC00312 group was significantly increased; (C, D) The expression of MMP9 in tumor tissues was detected by immunohistochemistry, showing that overexpression of LINC00312 reduced the percentage of MMP9 positive cells, and low expression increased the percentage of MMP9 positive cells; (E, F) The results of Western blotting showed that overexpression of LINC00312 reduced the expression of PI3K and p-Akt. * Compared with the blank group, $P<0.05$; \# compared with the si-control group, $P<0.05$. Results presented as the mean \pm SD with 3 independent experiments.

\section{Discussion}

TC is the most common endocrine malignancy, with increased incidence in many countries, and it accounts for about $0.5 \%$ of cancer deaths worldwide every year $[22,23]$. Thus, identification of new treatment methods for effectively inhibiting the growth and invasion of TC is needed. Mounting evidence proves that IncRNAs plays an important role in cancer pathogenesis [24,25]. In our study, we assessed the relationship between LINC00312 and TC, demonstrating that LINC00312 can act as a tumor suppressor in TC by attenuating the PI3K/Akt signaling pathway, and LINC00312 could be a novel diagnosis biomarker and a promising therapeutic target for TC patients. 
First, LINC00312 expression in TC cell lines and tissues were detected by qRT-PCR, and the results indicated that LINC00312 is expressed at low levels in TC cell lines and TC tissues. LINC00312 is a newly discovered IncRNA. To the best of our knowledge, only 5 studies have reported the specific role of LINC00312 in diseases and cancers, including nasopharyngeal carcinoma, non-small cell lung cancer, bladder cancer, and TC. Zhang et al. first revealed that expression of LINC00312 was significantly down-regulated in nasopharyngeal carcinoma tissues [26], and demonstrated that LINC00312 expression was positively correlated with lymph node metastasis but was negatively correlated with tumor size. A study focused on the role of LINC00312 in bladder cancer found lower expression of LINC00312 in bladder cancer tissues when compared with the adjacent normal tissues [27]. Additionally, lower expression of LINC00312 was also found in TC cells [13], which is consistent with our result. These findings show the important role of LINC00312 in cancers.

TC cell proliferation and invasion were also detected via CCK-8/EdU and Transwell assay, and the results revealed that proliferation and invasion abilities of TC cells were weakened after overexpression of LINC00312. Tumorigenesis and cancer progression can be caused by genetic factors and environmental exposure, as well as by epigenetic alteration, including histone modifications, DNA methylation, and regulation by miRNAs or IncRNAs [28]. Accumulating evidence has suggested a crucial role of IncRNAs in modulating the development of cancer through multiple pathogenic processes, including cell differentiation, proliferation, and invasion [29-32] NAG7, a newly-discovered putative tumor suppressor gene, was found to inhibit bladder cancer cell migration and invasion by its overexpression [27,33]. Low expression of CASC2 was found in TC, and overexpression of CASC2 inhibited the TC proliferation and arrested the cell cycle at G0/G1 stage in TC cells [34]. To investigate the biological function of LINC00312 in TC cells in vivo, we designed orthotopic TC xenografts in nude mice. The results also confirmed that overexpression of LINC00312 inhibited the proliferation and invasion of TC cells.

\section{References:}

1. Kapiteijn E, Schneider TC, Morreau H et al: New treatment modalities in advanced thyroid cancer. Ann Oncol, 2012; 23: 10-18

2. Schonfeld S J, Ron E, Kitahara C M et al: Hormonal and reproductive factors and risk of postmenopausal thyroid cancer in the NIH-AARP Diet and Health Study. Cancer Epidemiol, 2011; 35: e85-90

3. Xu L, Port M, Landi S et al: Obesity and the risk of papillary thyroid cancer: A pooled analysis of three case-control studies. Thyroid, 2014; 24: 966-74

4. Peterson E, De $P$, Nuttall R: BMI, diet and female reproductive factors as risks for thyroid cancer: A systematic review. PLoS One, 2012; 7: e29177

5. Pellegriti G, Frasca F, Regalbuto $C$ et al: Worldwide increasing incidence of thyroid cancer: Update on epidemiology and risk factors. J Cancer Epidemiol, 2013; 2013: 965212
More importantly, we found that overexpression of LINC00312 inhibited the activation of the PI3K/Akt signaling pathway in TC, and the role of MMP9 expression induced by overexpressed LINC00312 or si-LINC00312 could be weakened by LY294002. As a putative tumor suppressor gene, LINC00312 has been found to play a significant role in many cancers. Among the MMPs, MMP-9 was not only involved in extracellular matrix degradation during tissue remodeling, but also plays a significant role in pathological processes, including tumor invasion and metastasis $[35,36]$. MMP-9 has been shown to be highly expressed and to play an important role in the pathogenesis of TC [37-39]. Previous evidence demonstrated that many kinds of signaling pathways, such as PI3K, p38MAPK, and MAPK/ERK, were essential signaling pathways involved in the activation of MMP-9 $[39,40]$. It was also confirmed by previous studies that the tumor suppressor gene PTEN (a negative regulator of the PI3K/Akt signaling pathway), inhibited MMP9-induced cell invasion and significantly reduced MMP-9 promoter activity $[41,42]$. Thus, the relationship between MMP-9 and the PI3K/Akt pathway has been clarified. A recent study conducted by Liu et al. showed that LINC00312 can suppress TC cell invasion and tumorigenesis ability [13]. Therefore, we hypothesize that overexpression of LINC00312 inhibits the activation of the PI3K/Akt signaling pathway and then suppresses proliferation and invasion abilities of TC cells.

\section{Conclusions}

In conclusion, this study provides evidence that LINC00312 is significantly downregulated in TC, it can act as a tumor suppressor, and could be a novel diagnosis biomarker in TC by attenuating the $\mathrm{PI} 3 \mathrm{~K} / \mathrm{Akt}$ signaling pathway. Due to the complicate mechanism involved in TC and in the IncRNAs, further research is needed.

\section{Conflicts of interest}

None.

6. Anderson RT, Linnehan JE, Tongbram V et al: Clinical, safety, and economic evidence in radioactive iodine-refractory differentiated thyroid cancer: A systematic literature review. Thyroid, 2013; 23: 392-407

7. Nixon IJ, Whitcher MM, Palmer FL et al: The impact of distant metastases at presentation on prognosis in patients with differentiated carcinoma of the thyroid gland. Thyroid, 2012; 22: 884-89

8. Capdevila J, Iglesias L, Halperin I et al: Sorafenib in metastatic thyroid cancer. Endocr Relat Cancer, 2012; 19: 209-16

9. Wu XS, Wang XA, Wu WG et al: MALAT1 promotes the proliferation and metastasis of gallbladder cancer cells by activating the ERK/MAPK pathway. Cancer Biol Ther, 2014; 15: 806-14

10. Bao H, Guo CG, Qiu PC et al: Long non-coding RNA Igf2as controls hepatocellular carcinoma progression through the ERK/MAPK signaling pathway. Oncol Lett, 2017; 14: 2831-37 
11. Eyangoh S: Good data collection is essential for a better understanding of Buruli ulcer. Lancet Glob Health, 2014; 2: e371-72

12. Zhang W, Huang C, Gong Z et al: Expression of LINC00312, a long intergenic non-coding RNA, is negatively correlated with tumor size but positively correlated with lymph node metastasis in nasopharyngeal carcinoma. J Mol Histol, 2013; 44: 545-54

13. Liu K, Huang W, Yan DQ et al: Overexpression of long intergenic noncoding RNA LINC00312 inhibits the invasion and migration of thyroid cancer cells by down-regulating microRNA-197-3p. Biosci Rep, 2017; 37(4): pii: BSR20170109

14. Xing M: Genetic alterations in the phosphatidylinositol-3 kinase/Akt pathway in thyroid cancer. Thyroid, 2010; 20: 697-706

15. Liang F, Yue J, Wang J et al: GPCR48/LGR4 promotes tumorigenesis of prostate cancer via PI3K/Akt signaling pathway. Med Oncol, 2015; 32: 49

16. Liu X, Li Z, Song $Y$ et al: AURKA induces EMT by regulating histone modification through Wnt/beta-catenin and PI3K/Akt signaling pathway in gastric cancer. Oncotarget, 2016; 7: 33152-64

17. Liu R, Liu D, Trink E et al: The Akt-specific inhibitor MK2206 selectively inhibits thyroid cancer cells harboring mutations that can activate the PI3K/ Akt pathway. J Clin Endocrinol Metab, 2011; 96: E577-85

18. Liu D, Hou P, Liu Z et al: Genetic alterations in the phosphoinositide 3-kinase/Akt signaling pathway confer sensitivity of thyroid cancer cells to therapeutic targeting of Akt and mammalian target of rapamycin. Cancer Res, 2009; 69: 7311-19

19. Furuya F, Lu C, Willingham MC, Cheng SY: Inhibition of phosphatidylinositol 3-kinase delays tumor progression and blocks metastatic spread in a mouse model of thyroid cancer. Carcinogenesis, 2007; 28: 2451-58

20. Rothhut B, Ghoneim C, Antonicelli F, Soula-Rothhut M: Epidermal growth factor stimulates matrix metalloproteinase- 9 expression and invasion in human follicular thyroid carcinoma cells through Focal adhesion kinase. Biochimie, 2007; 89: 613-24

21. Volpe V, Raia Z, Sanguigno L et al: NGAL controls the metastatic potential of anaplastic thyroid carcinoma cells. J Clin Endocrinol Metab, 2013; 98: 228-35

22. Cho BY, Choi HS, Park YJ et al: Changes in the clinicopathological characteristics and outcomes of thyroid cancer in Korea over the past four decades. Thyroid, 2013; 23: 797-804

23. Liu L, Yang J, Zhu X et al: Long noncoding RNA H19 competitively binds miR-17-5p to regulate YES1 expression in thyroid cancer. Febs J, 2016; 283 2326-39

24. Silva VR, Secolin R, Vemuganti R et al: Acute liver failure is associated with altered cerebral expression profiles of long non-coding RNAs. Neurosci Lett, 2017; 656: 58-64

25. Ning S, Zhang J, Wang P et al: Lnc2Cancer: a manually curated database of experimentally supported IncRNAs associated with various human cancers. Nucleic Acids Res, 2016; 44: D980-85
26. Bienaime F, Girard D, Anglicheau D et al: Vitamin D status and outcomes after renal transplantation. J Am Soc Nephrol, 2013; 24: 831-41

27. Wang YY, Wu ZY, Wang GC et al: LINC00312 inhibits the migration and in vasion of bladder cancer cells by targeting miR-197-3p. Tumour Biol, 2016 37: 14553-63

28. Zheng $H$, Wang $M$, Jiang $L$ et al: BRAF-activated long noncoding RNA modulates papillary thyroid carcinoma cell proliferation through regulating thyroid stimulating hormone receptor. Cancer Res Treat, 2016; 48: 698-707

29. Ernst C, Morton CC: Identification and function of long non-coding RNA. Front Cell Neurosci, 2013; 7: 168

30. Maruyama R, Suzuki H. Long noncoding RNA involvement in cancer. BMB Rep 2012; 45: 604-11.

31. Esteller M: Non-coding RNAs in human disease. Nat Rev Genet, 2011; 12: 861-74

32. Gibb EA, Brown CJ, Lam WL: The functional role of long non-coding RNA in human carcinomas. Mol Cancer, 2011; 10: 38

33. Tan C, Li J, Xie Y et al: Preliminary function study of NAG7 using two-dimensional electrophoresis and mass spectrometry. Sheng Wu Hua Xue Yu Sheng Wu Wu Li Xue Bao (Shanghai), 2001; 33: 373-78

34. Xiong $X$, Zhu $H$, Chen $X$ : Low expression of long noncoding RNA CASC2 indicates a poor prognosis and promotes tumorigenesis in thyroid carcinoma. Biomed Pharmacother, 2017; 93: 391-97

35. Bjorklund $M$, Koivunen E: Gelatinase-mediated migration and invasion of cancer cells. Biochim Biophys Acta, 2005; 1755: 37-69

36. Bauvois B: New facets of matrix metalloproteinases MMP-2 and MMP-9 as cell surface transducers: Outside-in signaling and relationship to tumor progression. Biochim Biophys Acta, 2012; 1825: 29-36

37. Maeta $\mathrm{H}$, Ohgi $\mathrm{S}$, Terada T: Protein expression of matrix metalloproteinases 2 and 9 and tissue inhibitors of metalloproteinase 1 and 2 in papillary thyroid carcinomas. Virchows Arch, 2001; 438: 121-28

38. Baldini E, Toller M, Graziano FM et al: Expression of matrix metalloprotein ases and their specific inhibitors in normal and different human thyroid tumor cell lines. Thyroid, 2004; 14: 881-88

39. Lin SY, Wang YY, Sheu WH: Preoperative plasma concentrations of vascular endothelial growth factor and matrix metalloproteinase 9 are associated with stage progression in papillary thyroid cancer. Clin Endocrinol (Oxf), 2003; 58: 513-18

40. Nishikawa M, Nishiguchi S, Shiomi S et al: Somatic mutation of mitochondrial DNA in cancerous and noncancerous liver tissue in individuals with hepatocellular carcinoma. Cancer Res, 2001; 61: 1843-45

41. Park MJ, Kim MS, Park IC et al: PTEN suppresses hyaluronic acid-induced matrix metalloproteinase- 9 expression in U87MG glioblastoma cells through focal adhesion kinase dephosphorylation. Cancer Res, 2002; 62: 6318-22

42. Moon SK, Kim HM, Kim CH: PTEN induces $\mathrm{G} 1$ cell cycle arrest and inhibits MMP-9 expression via the regulation of NF-kappaB and AP-1 in vascular smooth muscle cells. Arch Biochem Biophys, 2004; 421: 267-76 